\title{
Comments on Two-Loop Kac-Moody Algebras
}

\author{
L.A. Ferreira円, J.F. Gomes ${ }^{1}$ and A.H. Zimerman ${ }^{1}$ \\ Instituto de Física Teórica-UNESP \\ Rua Pamplona 145 \\ 01405 São Paulo, Brazil
}

A. Schwimmere阿

SISSA and INFN, Sezione de Trieste

2-4 via Beirut

Trieste 34014, Italy

\begin{abstract}
It is shown that the two-loop Kac-Moody algebra is equivalent to a two variable loop algebra and a decoupled $\beta-\gamma$ system. Similarly WZNW and CSW models having as algebraic structure the Kac-Moody algebra are equivalent to an infinity of versions of the corresponding ordinary models and decoupled abelian fields.
\end{abstract}

\footnotetext{
${ }^{1}$ Supported in part by CNPq; e-mail: 47553::LAF, 47553::JFG

${ }^{2}$ On leave from Department of Physics, Weizmann Institute, Rehovot, 76100 Israel

${ }^{3}$ Supported by FAPESP-Brazil and BSF
} 
Infinite dimensional extensions of the chiral algebras appearing in Conformal Field Theories received a lot of attention recently. The general feature of these extensions is the dependence of the currents on an additional variable besides $z$, the chiral coordinate on the 2-D world sheet. These new algebras [1] include the different versions of $W_{\infty}$ [2, 3] and the two loop Kac-Moody $(\mathrm{KM})$ 田, 5, 6, 7, 8].

One version of the two loop K-M algebra was introduced in ref. [4] in the context of a WZNW type of model having as target space an infinite dimensional group manifold which, under a Hamiltonian reduction procedure, gives rise to the conformal affine Toda model (CAT) [9]. Under a similar line of reasoning a Chern-Simons theory associated to the same algebraic structure was also considered in ref. [7].

For the $W_{\infty}$ type algebra it was pointed out by Witten 10 that there is a natural geometric interpretation in terms of symplectic diffeomorphisms of the cotangent bundle $T^{*} \Sigma$ of a Riemann surface $\Sigma$. The second variable is therefore intrinsically linked to $z$. Such an interpretation is lacking for two-loop KM algebras. We suspect that in this case the two variables can be decoupled. The main result of this paper is to show that this is indeed true. The two-loop KM algebra can be decomposed by a redefinition of currents into a two variable loop algebra, with two central extensions, and a $\beta$ - $\gamma$ system.

As a reflection of this algebraic fact the WZNW and CSW models based on KM algebras are shown to be equivalent to an infinite number of copies, labeled by an additional variable, of the ordinary (based on Lie algebra) versions of the corresponding models and decoupled sets of abelian fields.

Let us start with the algebraic treatment. The two-loop KM algebra is defined by the following commutation relations 4

$$
\begin{aligned}
{\left[J_{a}^{m}(x), J_{b}^{n}(y)\right] } & =i f_{a b}^{c} J_{c}^{m+n}(x) \delta(x-y)+i \frac{k}{2 \pi} g_{a b} \partial_{x} \delta(x-y) \delta_{m,-n}+C(x) \delta(x-y) g_{a b} m \delta_{m,-n} \\
{\left[D(x), J_{a}^{m}(y)\right] } & =m J_{a}^{m}(y) \delta(x-y) \\
{[C(x), D(y)] } & =i \frac{k}{2 \pi} \partial_{x} \delta(x-y) \\
{\left[C(x), J_{a}^{m}(y)\right] } & =0
\end{aligned}
$$

where $f_{a b}^{c}$ are the structure constants of a finite semisimple Lie algebra $\mathcal{G}$, while $g_{a b}$ is the Killing form of $\mathcal{G}\left(g_{a b}=\operatorname{Tr}\left(T_{a} T_{b}\right), T_{a}\right.$ being the generator of $\left.\mathcal{G}\right)$ and $n, m \in \mathbb{Z}$.

We will consider (1)-(4) as the algebra of equal time commutators of the currents with the spatial variable $x$ being on a circle of radius 1 , and the currents satisfying the periodic boundary conditions $J_{a}^{m}(x+2 \pi)=J_{a}^{m}(x), C(x+2 \pi)=C(x)$ and $D(x+2 \pi)=D(x)$.

Using the fact that $C(x)$ commutes with $J_{a}^{m}(x)$ we can define a new set of currents by

$$
\tilde{J}_{a}^{m}(x)=J_{a}^{m}(x) \exp \left(\frac{m}{k} \sum_{r \neq 0} \frac{C_{r}}{r} e^{-i r x}\right)
$$

where $C_{r}$ are the modes of $C(x)$, i.e.

$$
C(x)=\frac{1}{2 \pi} \sum_{r=-\infty}^{\infty} C_{r} e^{-i r x}
$$


The zero mode $C_{0}$ is not included in the transformation (5) in orde $\mathrm{r}$ to respect the periodicity properties of $\tilde{J}_{a}^{m}(x)$. Notice that the transformation (5) is defined on a given representation and not on the abstract algebra. Using (11)-(侜) and the identity

$$
f(x) \partial_{x} \delta(x-y)=f(y) \partial_{x} \delta(x-y)-\partial_{y} f(y) \delta(x-y)
$$

one obtains

$$
\begin{aligned}
{\left[\tilde{J}_{a}^{m}(x), \tilde{J}_{b}^{n}(y)\right] } & =i f_{a b}^{c} \tilde{J}_{c}^{m+n}(x) \delta(x-y)+i \frac{k}{2 \pi} g_{a b} \partial_{x} \delta(x-y) \delta_{m,-n}+\frac{C_{0}}{2 \pi} \delta(x-y) g_{a b} m \delta_{m,-n} \\
{\left[D_{0}, \tilde{J}_{a}^{m}(x)\right] } & =m \tilde{J}_{a}^{m}(x) \\
{\left[D_{r}, \tilde{J}_{a}^{m}(x)\right] } & =0 \quad r \neq 0
\end{aligned}
$$

where $D_{r}$ are the modes of $D(x)$, i.e. , $D(x)=\frac{1}{2 \pi} \sum_{r=-\infty}^{\infty} D_{r} e^{-i r x} . D_{0}$ is the operator measuring the "momenta" $m$ in an usual KM algebra [1]. Formulae (8)-(10) show that the $C-D$ system is decoupled from the $\tilde{J}^{\prime}$ s.Introducing $\bar{x}$ as a conjugate variable to $m, \tilde{J}(x, \bar{x})$ obeys an algebra (Eq.(8)) completely symmetric in $x, \bar{x} . C_{0}$ commutes with all the operators, i.e., acts like a second central extension in the algebra of $\tilde{J}^{\prime} s$.

For certain classes of representations one of the central extensions can be removed as we now show. In order to do that we have to make specific assumptions about the groups and classes of representations. We study algebras based on a compact Lie group and we limit ourselves to unitary, highest weight representations for the $\tilde{J}_{a}^{m}(x)$ currents (we will discuss the possibility for the decoupled $D-C$ system afterwards). Decomposing $\tilde{J}_{a}^{m}(x)$ in its modes as $\tilde{J}_{a}^{m}(x)=$ $\frac{1}{2 \pi} \sum_{r=-\infty}^{\infty} \tilde{J}_{a}^{m, r} e^{-i r x}$, we see from (8) that we can require the highest weight state to be annihilated by all the operators corresponding to $(m, r)$ in a half plane limited by $m C_{0}+r k>0$. Then we can use a slight extension of the standard argument[11] leading to the quantization of $k$. Consider the $S U(2)$ subalgebras generated by (Weyl-Cartan basis)

$$
E_{\alpha}^{1,0} \quad ; \quad E_{-\alpha}^{-1,0} \quad ; \quad \frac{2}{\alpha^{2}}\left(\alpha \cdot H^{0,0}+k\right)
$$

and

$$
E_{\alpha}^{0,1} \quad ; \quad E_{-\alpha}^{0,-1} \quad ; \quad \frac{2}{\alpha^{2}}\left(\alpha \cdot H^{0,0}+C_{0}\right)
$$

The unitary, highest weight representations of the algebra (8-10) will break into unitary, highest weight representations of these subalgebras. Since $\frac{2}{\alpha^{2}}\left(\alpha \cdot H^{0,0}+k\right)$ and $\frac{2}{\alpha^{2}}\left(\alpha \cdot H^{0,0}+C_{0}\right)$ play the role of $2 T_{3}$, their eigenvalues must be integers. But since for a given weight $\mu, 2 \mu . \alpha / \alpha^{2}$ is also an integer, one concludes that $k$ and $C_{0}$ must be integers (when the squared length of the highest root is normalized to 2). Such an integral $C_{0}$ can be removed from the algebra, as we now show.

Consider the relabeling of the pair of indices $(m, r)$ defined by

$$
\left(\begin{array}{c}
m^{\prime} \\
r^{\prime}
\end{array}\right)=M\left(\begin{array}{c}
m \\
r
\end{array}\right)
$$

where $M=\left(\begin{array}{ll}a & b \\ c & d\end{array}\right) ; a, b, c, d \in \mathbb{Z}$ and $\operatorname{det} M=1$. The last condition is needed in order to make the transformation one to one. We define then new currents by

$$
\tilde{j}_{a}^{m, r}=\tilde{J}_{a}^{m^{\prime}, r^{\prime}}
$$


Using (8) and (13) the new currents satisfy the algebra

$$
\left[\tilde{j}_{a}^{m, r}, \tilde{j}_{b}^{n, s}\right]=i f_{a b}^{c} \tilde{j}_{c}^{m+n, r+s}+k^{\prime} g_{a b} r \delta_{r+s, 0} \delta_{m+n, 0}+C^{\prime}{ }_{0} g_{a b} m \delta_{m+n, 0} \delta_{r+s, 0}
$$

with

$$
\left(\begin{array}{ll}
C^{\prime}{ }_{0} & k^{\prime}
\end{array}\right)=\left(\begin{array}{ll}
C_{0} & k
\end{array}\right) M
$$

and where we have used the fact that $\delta_{m^{\prime}+n^{\prime}, 0} \delta_{r^{\prime}+s^{\prime}, 0}=\delta_{m+n, 0} \delta_{r+s, 0}($ if $\operatorname{det} M \neq 0)$.

We discuss separately the two possibilities:

1. $k$ is a divisor of $C_{0}$. So one can choose $a=1, b=0, c=-C_{0} / k$ and $d=1$. Then $k^{\prime}=k$ and $C^{\prime}{ }_{0}=0$. Analogously if $C_{0}$ is a divisor of $k$ one can choose $a=k / C_{0}, b=1, c=-1$ and $d=0$. Then one gets $k^{\prime}=C_{0}$ and again $C^{\prime}{ }_{0}=0$.

2. If $C_{0} / k$ is rational, i.e., $C_{0}=l p$ and $k=l q$ with $(p, q)$ relative primes, one chooses $a=q$ and $c=-p$ with $b$ and $d$ satisfying $d q+b p=1$, which always has a solution. One then gets $k^{\prime}=l$ and $C^{\prime}{ }_{0}=0$.

Consequently the last term on the r.h.s. of (15) can always be transformed away reducing (15) to a very similar structure as given in [5].

Introducing the currents

$$
j_{a}^{r}(\bar{x}) \equiv \frac{1}{2 \pi} \sum_{m=-\infty}^{\infty} \tilde{j}_{a}^{m, r} e^{i m \bar{x}}
$$

one can then write (15) as

$$
\left[j_{a}^{r}(\bar{x}), j_{b}^{s}(\bar{y})\right]=\tilde{\delta}(\bar{x}-\bar{y})\left(i f_{a b}^{c} j_{c}^{r+s}(\bar{x})+\frac{k^{\prime}}{2 \pi} g_{a b} r \delta_{r+s, 0}\right)
$$

where $\tilde{\delta}(\bar{x}-\bar{y})=\frac{1}{2 \pi} \sum_{m=-\infty}^{\infty} e^{i m(\bar{x}-\bar{y})}=\sum_{n=-\infty}^{\infty} \delta(\bar{x}-\bar{y}+2 \pi n)$. This indicates that the algebra is decomposed into an infinity of decoupled ordinary $\mathrm{KM}$ algebras and a decoupled $D-C$ system (except for their zero modes which are still coupled to the ordinary KM's, having the role mentioned above).

The highest weight representation of (18) is constructed by applying $j_{a}^{r}(\bar{x})$ on the highest weight state $\mid \lambda(\bar{x})>$ characterized by

$$
\begin{aligned}
& j_{a}^{r}(\bar{x}) \mid \lambda(\bar{y})>=0 ; \quad r>0 \\
& j_{\alpha}^{0}(\bar{x}) \mid \lambda(\bar{y})>=0
\end{aligned}
$$

where $\alpha$ is a positive root of the underlying finite Lie algebra, and

$$
j_{H_{i}}^{0}(\bar{x})\left|\{\lambda(\bar{y})\}>=\lambda_{i}(\bar{x})\right|\{\lambda(\bar{y})\}>
$$

where $j_{H_{i}}^{0}(\bar{x})$ correspond to the Cartan subalgebra generators. The "distribution of weights" $\lambda(\bar{x})$ is arbitrary, being limited by the condition

$$
\int \lambda(\bar{x}) d \bar{x} \equiv \lambda
$$

where $\lambda$ is a weight of the ordinary Lie algebra.

For the decoupled $D-C$ system we have two options 
1. A unitary representation, which however will not be highest weight, in terms of the modes of a scalar field $\varphi, C(x)$ being identified with $\partial_{x} \varphi$ and $D(x)$ with $\Pi_{\varphi}$ (the conjugate momentum).

2. A non unitary highest weight representation in terms of a $\beta-\gamma$ system [14, $C(x)$ being identified with $\partial_{x} \beta$ and $D(x)$ with $\gamma$.

We remark that the delta function normalization in (18) shows that irreducible representations (irreps) of that algebra can not be constructed by just taking infinite products of ordinary KM irreps. One can convince oneself of that by considering the algebra (15) (with $C_{0}^{\prime}=0$ ) with the integers $m$ and $n$ varying from $-N$ to $N$ and the sum modulo $2 N+1$. Making a discret version of the transformation (17) one obtains (18) with a Kronecker delta and central term $\frac{k^{\prime}}{2 N+1}$. Therefore by taking the limit $N \rightarrow \infty$ one sees (15) is equivalent to an infinity of ordinary KM algebras with vanishingly small central extension.

We now show that the algebraic decomposition discussed above can be implemented in the WZNW model having as current algebra the two loop KM [4]. The arguments are not entirely rigorous but show the essential features of the factorization.

The ordinary Lie algebra underlying the standard WZNW model is replaced by a KM algebra with commutations relations

$$
\begin{aligned}
{\left[T_{a}^{m}, T_{b}^{n}\right]=} & i f_{a b}^{c} T_{c}^{m+n}+\hat{c} g_{a b} m \delta_{m+n, 0} \\
{\left[\hat{d}, T_{a}^{m}\right]=} & m T_{a}^{m} \\
{[\hat{c}, \hat{d}]=0 \quad } & {\left[\hat{c}, T_{a}^{m}\right]=0 }
\end{aligned}
$$

and trace form

$$
\begin{array}{r}
\operatorname{Tr}\left(T_{a}^{m} T_{b}^{n}\right)=g_{a b} ; \delta_{m+n, 0} \operatorname{Tr}(\hat{c} \hat{d})=1 \\
\operatorname{Tr}(\hat{d} \hat{d})=\operatorname{Tr}(\hat{c} \hat{c})=\operatorname{Tr}\left(\hat{c} T_{a}^{m}\right)=\operatorname{Tr}\left(\hat{d} T_{a}^{m}\right)=0
\end{array}
$$

Defining formally a group element $\hat{g}$ by

$$
\hat{g}=\exp \left(i \sum_{m} T_{a}^{m} \alpha_{-m}^{a}+i \beta \hat{c}+i \gamma \hat{d}\right)
$$

The WZNW action is defined by

$$
S\left(\hat{g}\left(x_{+}, x_{-}\right)\right) \equiv k\left(\frac{1}{4 \pi} \int_{\Sigma} \operatorname{Tr}\left(\hat{g}^{-1} \partial_{+} \hat{g} \hat{g}^{-1} \partial_{-} \hat{g}\right)+\frac{1}{12 \pi} \int_{B} \operatorname{Tr}\left(\hat{g}^{-1} d \hat{g}\right)^{3}\right)
$$

Since the global properties of the group defined by (26) are not known it is not clear at this stage if $k$ should be quantized and what are the conditions on the zero modes of $\alpha, \beta$, and $\gamma$ which would make $\hat{g}$ single valued on the two dimensional manifold $\Sigma$. The action (27) leads to the conservation of the left and right handed currents $\partial_{-} \hat{g} \hat{g}^{-1}$ and $\hat{g}^{-1} \partial_{+} \hat{g}$. Their Poisson brackets lead to the "two-loop affine algebra" for $\hat{J}(z)=\sum_{m, a, b} g^{a b} J_{a}^{m}(z) T_{b}^{-m}+D(z) \hat{c}+C(z) \hat{d}$ where $z \in \Sigma$.

In order to facilitate some of the manipulations which will follow it is convenient to present the algebra as equal time commutators with spatial variable $x$ being on a circle of radius 1 (i.e. 
$\left.z=e^{i x}\right)$ and the discrete index $m$ with its conjugate variable $\bar{x} \in(0,2 \pi)$. This amounts to defining

$$
J_{a}(x, \bar{x}) \equiv \frac{1}{2 \pi} \sum_{m=-\infty}^{\infty} J_{a}^{m}(x) e^{-i m \bar{x}}
$$

The two-loop KM algebra (1)-(雨) will be then

$$
\begin{aligned}
{\left[J_{a}(x, \bar{x}), J_{b}(y, \bar{y})\right]=} & i f_{a b}^{c} J_{c}(x, \bar{x}) \delta(x-y) \delta(\bar{x}-\bar{y})+i k g_{a b} \partial_{x} \delta(x-y) \delta(\bar{x}-\bar{y}) \\
& +C(x) \delta(x-y) g_{a b} \partial_{\bar{x}} \delta(\bar{x}-\bar{y}) \\
{\left[D(x), J_{a}(y, \bar{y})\right]=} & i \partial_{\bar{y}} J_{a}(y, \bar{y}) \delta(x-y) \\
{[C(x), D(y)]=} & i k \partial_{x} \delta(x-y) \\
{\left[C(x), J_{a}(y, \bar{y})\right]=} & 0
\end{aligned}
$$

We return now to the action (27). A general group element $\hat{g}$ can be factorized, using the Baker-Campbell-Hausdorff theorem as follows

$$
\begin{aligned}
\hat{g} & \equiv \exp \left(i \int d \bar{x} T_{a}(\bar{x}) \alpha^{a}(\bar{x})+i \beta \hat{c}+i \gamma \hat{d}\right) \\
& =\exp \left(i \int d \bar{x} T_{a}(\bar{x}) \rho^{a}(\bar{x})\right) \exp (i \bar{\beta} \hat{c}+i \gamma \hat{d}) \equiv \hat{g}_{1} \hat{g}_{2}
\end{aligned}
$$

where $\rho^{a}(\bar{x})$ is the composite parameter, in the ordinary Lie group sense (at each point $\bar{x}$ separately) of the parameters $\sigma^{a}(\bar{x})$ and $\sigma^{a}(\bar{x}-\gamma) ; \sigma^{a}$ being the solution of the differential equation

$$
\partial_{\bar{x}} \sigma(\bar{x})=\frac{M(\bar{x})}{\gamma\left(1-e^{M(\bar{x})}\right)} \alpha(\bar{x})
$$

with the matrix $M(\bar{x})$ being defined as

$$
M_{a}^{c}(\bar{x})=f_{a b}^{c} \alpha^{b}(\bar{x})
$$

We do not reproduce the rather involved expression of $\bar{\beta}$ in terms of $\alpha, \beta, \gamma$ which is not essential for our argument. We use now the Polyakov-Wiegmann identity[15]

$$
S(\hat{g})=S\left(\hat{g}_{1}\right)+S\left(\hat{g}_{2}\right)+\frac{1}{2 \pi} \int_{\Sigma} \operatorname{Tr}\left(\partial_{-} \hat{g}_{2} \hat{g}_{2}^{-1} \hat{g}_{1}^{-1} \partial_{+} \hat{g}_{1}\right)
$$

Notice that $\partial_{-} \hat{g}_{2} \hat{g}_{2}^{-1}$ is a linear combination of the $\hat{d}$ and $\hat{c}$ generators. Although $\hat{g}_{1}$ is an exponential of a linear combination of $T_{a}(\bar{x})^{\prime} s$ only, $\hat{g}_{1}^{-1} \partial_{+} \hat{g}_{1}$ contains terms also in the direction of $\hat{c}$. So, according to (25), the last term on the r.h.s. of (36) apparently does not vanish. However we can make it to vanish as follows. Write

$$
i \hat{g}_{1}^{-1} \partial_{\mu} \hat{g}_{1}=\Gamma_{\mu} \hat{c}+\ldots
$$

Since $\Gamma_{\mu}$ is a vector in $2-\mathrm{D}$ one can write it as

$$
\Gamma_{\mu}=\partial_{\mu} A+\epsilon_{\mu \nu} \partial^{\nu} B
$$


Redefining $\hat{g}$ in (33) as $\hat{g}=\hat{g}_{1} \hat{g}_{2}=\hat{g}_{1}^{\prime} \hat{g}_{2}^{\prime}$ with

$$
\hat{g}_{1}^{\prime}=e^{i(A-B) \hat{c}} \hat{g}_{1} ; \hat{g}_{2}^{\prime}=e^{-i(A-B) \hat{c}} \hat{g}_{2}
$$

one gets

$$
\begin{aligned}
i \hat{g}_{1}^{\prime-1} \partial_{\mu} \hat{g}_{1}^{\prime} & =i \hat{g}_{1}^{-1} \partial_{\mu} \hat{g}_{1}-\partial_{\mu}(A-B) \hat{c} \\
& =\left(\partial_{\mu} B+\epsilon_{\mu \nu} \partial^{\nu} B\right) \hat{c}+\ldots
\end{aligned}
$$

It then follows that $\hat{g}_{1}^{\prime-1} \partial_{+} \hat{g}_{1}^{\prime} \sim \hat{g}_{1}^{\prime-1} \partial_{0} \hat{g}_{1}^{\prime}+\hat{g}_{1}^{\prime-1} \partial_{1} \hat{g}_{1}^{\prime}$ does not contain any term in the direction of $\hat{c}$. Consequently the overlap term in (36) will vanish. Moreover due to the properties of the trace form (25), $S\left(\hat{g}_{1}^{\prime}\right)$ does not get any contribution of terms in the direction of $\hat{c}$ and therefore it could be equivalently generated by an algebra of $T_{a}^{m}$ with $\hat{c}$ (and also $\hat{d}$ ) put to zero (so called loop algebra, and which is consistent with the Jacobi identities being a direct product of decoupled ordinary Lie algebras at each point $\bar{x})$. Finally $S\left(\hat{g}_{2}\right)$ is simply

$$
S\left(\hat{g}_{2}\right)=\frac{k}{4 \pi} \int d^{2} x \partial_{\mu} \gamma \partial^{\mu} \bar{\beta}
$$

i.e., a pair of scalar fieds coupled off-diagonally.

The current algebra that the two-loop decoupled actions (36) would lead to is therefore

$$
\begin{aligned}
{\left[j_{a}(x, \bar{x}), j_{b}(y, \bar{y})\right] } & =\delta(\bar{x}-\bar{y})\left(i f_{a b}^{c} j_{c}(x, \bar{x})+i k g_{a b} \partial_{x} \delta(x-y)\right) \\
{[\tilde{C}(x), \tilde{D}(y)] } & =i k \partial_{x} \delta(x-y)
\end{aligned}
$$

all other commutators, including $\left[\tilde{D}, j_{a}\right]$ vanishing.

Now, since (27) and (36) represent the same model, we expect the algebras (29)-(32) and (42) to be equivalent. As we mentioned before the above argument is not rigorous since the measure is not under control and the zero modes may be shared between $\hat{g}_{1}$ and $\hat{g}_{2}$ leading to a failure of the factorization.

Let us now study the factorization from the point of view of Chern-Simons-Witten (CSW) Lagrangeans. As discussed in ref. 12, 13 a three dimensional CSW Lagrangean based on a Lie algebra $\mathcal{G}$ has a gauge invariant Hilbert space related to the Hilbert space of the two-dimensional WZNW model built on $\mathcal{G}$ and therefore to the representation of the current algebra $\hat{\mathcal{G}}$. Motivated by this connection, in ref. [7] a CSW Lagrangean based on the KM algebra (22)-(24) was proposed. The gauge fields associated with $T_{a}^{m}, \hat{c}$ and $\hat{d}$ respectively are $\left(A_{\mu}\right)_{m}^{a}, B_{\mu}$ and $C_{\mu}$. It is convenient to replace the discrete index $m$ by a continuous periodic variable $\bar{x} \in(0,2 \pi)$ through

$$
A_{\mu}^{a}(x, \bar{x})=\sum_{m \in \mathbb{Z}}\left(A_{\mu}(x)\right)_{m}^{a} e^{i m \bar{x}}
$$

Then the CSW action proposed in [7] can be rewritten as

$$
S=\frac{1}{2 \pi} \int_{M \otimes S^{1}} d^{3} x d \bar{x} \epsilon^{\mu \nu \rho}\left(A_{\mu}^{a} \partial_{\nu} A_{\rho}^{a}+\frac{1}{3} f_{a b c} A_{\mu}^{a} A_{\nu}^{b} A_{\rho}^{c}+B_{\mu}\left(\partial_{\nu} C_{\rho}-\partial_{\rho} C_{\nu}\right)+C_{\mu} \frac{\partial A_{\nu}^{a}}{\partial \bar{x}} A_{\rho}^{a}\right)
$$


where $M$ is a three dimensional manifold. The gauge fields $A_{\mu}^{a}(x, \bar{x})$ should therefore be single valued on $M \otimes S^{1}$. In the generating functional $Z$, which is given by

$$
Z=\int d A d B d C e^{i k S}
$$

the integration over B leads to the delta function:

$$
\delta\left(\partial_{\nu} C_{\rho}-\partial_{\rho} C_{\nu}\right)
$$

This implies that

$$
C_{\rho}=\partial_{\rho} \alpha+\frac{\theta_{\rho}}{k}
$$

where $\theta_{\rho}$ are constants and $\alpha(x)$ is single valued on M. Inside the generating functional (45) the action $k S$ takes the form

$$
\frac{k}{2 \pi} \int d^{3} x d \bar{x} \epsilon^{\mu \nu \rho}\left(A_{\mu}^{a} \partial_{\nu} A_{\rho}^{a}+\frac{1}{3} f_{a b c} A_{\mu}^{a} A_{\nu}^{b} A_{\rho}^{c}+\phi a_{\mu} \alpha \frac{\partial A_{\nu}^{a}}{\partial \bar{x}} A_{\rho}^{a}+\frac{\theta_{\mu}}{k} \frac{\partial A_{\nu}^{a}}{\partial \bar{x}} A_{\rho}^{a}\right)
$$

Performing now the transformation

$$
\left(\bar{A}_{\mu}(x)\right)_{m}^{a}=\left(A_{\mu}(x)\right)_{m}^{a} e^{m \alpha(x)}
$$

we obtain for the generating functional (45)

$$
Z=\int d \bar{A} d \theta e^{i k \bar{S}} \int d B d \tilde{C} e^{i k \int d^{3} x \epsilon^{\mu \nu \rho} B_{\mu}\left(\partial_{\nu} \tilde{C}_{\rho}-\partial_{\rho} \tilde{C}_{\nu}\right)}
$$

where $\tilde{C}_{\mu}$ stands for the $C_{\mu}$ field whithout the zero mode $\theta_{\mu}$ and where we have reinserted the integration over $B_{\mu}$

$$
k \bar{S}=\frac{k}{2 \pi} \int d^{3} x d \bar{x} \epsilon^{\mu \nu \rho}\left(\bar{A}_{\mu}^{a} \partial_{\nu} \bar{A}_{\rho}^{a}+\frac{1}{3} f_{a b c} \bar{A}_{\mu}^{a} \bar{A}_{\nu}^{b} \bar{A}_{\rho}^{c}\right)+\frac{\theta_{\mu}}{2 \pi} \int d^{3} x d \bar{x} \epsilon^{\mu \nu \rho} \frac{\partial \bar{A}_{\nu}^{a}}{\partial \bar{x}} \bar{A}_{\rho}^{a}
$$

Notice that the Jacobian of the transformation (49) is one. We have therefore shown that under the above transformations the generating functional factorizes into two parts, one containing the $B-\tilde{C}$ system and the other the $\bar{A}-\theta$ system.

Let us now consider the transformation properties of $\bar{S}$ under the gauge transformation

$$
\bar{A}_{\mu}(x, \bar{x}) \rightarrow g^{-1}(x, \bar{x}) \bar{A}_{\mu} g(x, \bar{x})+g^{-1} \partial_{\mu} g+\frac{\theta_{\mu}}{k} g^{-1} \frac{\partial g(x, \bar{x})}{\phi a \bar{x}}
$$

Actually the action (51) is automatically invariant under the infinitesimal form of (52). Under the full finite transformation one gets the additional terms

$$
\begin{aligned}
& \frac{k}{12 \pi} f_{a b c} \int_{M \otimes S^{1}} d^{3} x d \bar{x} \epsilon^{\mu \nu \rho}\left(g^{-1} \partial_{\mu} g\right)^{a}\left(g^{-1} \partial_{\nu} g\right)^{b}\left(g^{-1} \partial_{\rho} g\right)^{c} \\
& +\frac{\theta_{\mu}}{4 \pi} f_{a b c} \int_{M \otimes S^{1}} d^{3} x d \bar{x} \epsilon^{\mu \nu \rho}\left(g^{-1} \frac{\partial g}{\partial \bar{x}}\right)^{a}\left(g^{-1} \partial_{\nu} g\right)^{b}\left(g^{-1} \partial_{\rho} g\right)^{c}
\end{aligned}
$$


If $g$ represents a nontrivial mapping from $M$ to the Lie group $G$ the first integral does not vanish and in order that the path integral will be invariant under $G$, we need to have $k$ quantized, i.e. $k \in \mathbb{Z}$. In a similar way choosing $M=S^{1}\left(x_{1}\right) \otimes M_{23}$ if $g\left(x_{\mu}, \bar{x}\right)$ is a nontrivial mapping from the manifold $M_{23} \otimes S^{1}(\bar{x})$ to $G$ we also need to quantize $\theta_{\mu}$, i.e. $\theta_{\mu} \in \mathbb{Z}$. Therefore $k$ and $\theta_{\mu}$ should be integers. The situation becomes particularly symmetric on a torus. We shall now proceed in steps. First we perform a linear reparametrization involving only coordinates $x^{\mu}$ by a matrix $N$ with integral coefficients and determinant one. Under such a transformation the first two terms in (51) are invariant, while the third term induces a transformation on $\theta_{\mu}$ i.e.

$$
\theta_{\mu}^{\prime}=\left(N^{-1}\right)_{\mu}^{\nu} \theta_{\nu}
$$

Using this transformation, $\theta_{\mu}$ can be oriented along one of the directions, e.g. $\theta_{1} \neq 0, \theta_{2}=\theta_{3}=0$. We next define a transformation of coordinates

$$
\left(\begin{array}{c}
x^{\prime 1} \\
\bar{x}^{\prime}
\end{array}\right)=\left(\begin{array}{ll}
a & b \\
c & d
\end{array}\right)\left(\begin{array}{c}
x^{1} \\
\bar{x}
\end{array}\right) \quad ; \quad x^{\prime 2}=x^{2}, x^{\prime 3}=x^{3}
$$

with integral parameters $a, b, c, d$ and unit determinant. If $k$ and $\theta_{1}$ have a maximal common divisor $l$, i.e. $k=l \bar{k}$ and $\theta_{1}=l \bar{\theta}_{1}$ with $\bar{k}$ and $\bar{\theta}_{1}$ being relatively primes, we define

$$
\begin{gathered}
\tilde{A}_{1}\left(x_{\mu}, \bar{x}\right)=\bar{k} \bar{A}_{1}\left(x_{\mu}^{\prime}, \bar{x}^{\prime}\right) \\
\tilde{A}_{2,3}\left(x_{\mu}, \bar{x}\right)=\bar{A}_{2,3}\left(x_{\mu}^{\prime}, \bar{x}^{\prime}\right)
\end{gathered}
$$

with $a=\bar{k}, b=\bar{\theta}_{1}$ and $c, d$ are integral solutions of $\bar{k} d-\bar{\theta}_{1} c=1$ which always exist. Then the action (51) can be rewritten as

$$
S=\frac{l}{2 \pi} \int d^{3} x d \bar{x} \epsilon^{\mu \nu \rho}\left(\tilde{A}_{\mu}^{a} \partial_{\nu} \tilde{A}_{\rho}^{a}+\frac{1}{3} f_{a b c} \tilde{A}_{\mu}^{a} \tilde{A}_{\nu}^{b} \tilde{A}_{\rho}^{c}\right)
$$

i.e. the standard CSW model with $\bar{x}$ labelling decoupled gauge fields. A strange feature of the action (58) is the summation over the coupling constant $l$ due to its implicit dependence on the $\theta_{\mu}$ which are dynamical degrees of freedom.

The decoupled nature of (58) proves again our conjecture, namely, the gauge invariant Hilbert space corresponding to (48) is related to the Hilbert space of decoupled WZNW models. Therefore the Hilbert space of the KM WZNW model in its original formulation (44) is isomorphic to the Hilbert space of decoupled ordinary WZNW models.

Finally let us comment on the possible applications of the above constructions to the study of conformal models. As shown in ref. 四 the CAT model can be obtained by Hamiltonian reduction from the WZNW model based on KM algebras. It will be very interesting to investigate the consequences of the factorization of the two-loop KM algebra to the CAT model. This could perhaps lead to a better understanding of CAT models and its relantionship to ordinary Toda field theories.

Acknowledgements One of us (A.S.) would like to thank IFT-UNESP for the warm hospitality extended to him. 


\section{References}

[1] E. Floratos and J. Iliopoulos, Phys. Lett. 201B (1988) 237.

[2] I. Bakas, Phys. Lett. 228B (1989) 57.

[3] C. Pope, L. Romans and X. Shen, Phys. Lett. 236B (1990) 173; ibid 245B (1990) 72.

[4] H. Aratyn, L.A. Ferreira, J.F. Gomes and A.H. Zimerman, Phys. Lett. 254B (1991) 372

[5] J.M. Maillet Phys. Lett. 167B, (1986), 401.

[6] A. Gerasimov, D. Lebedev and A. Morozov, Inter. J. Mod. Phys. A6 (1991) 977; A. Morozov, Sov. J. Nucl. Phys. 52 (1990) 755.

[7] L. Bonora, M. Martellini, Y.-Z. Zhang, Phys. Lett. 253B (1991) 373. L. Bonora, M. Martellini, Y.-Z. Zhang, Affine CS Theories and WZNW models, preprint SISSA/ISAS79/91/EP BONN-HE-91-08

[8] H. Aratyn, C.P. Constantinidis, L.A.Ferreira, J.F. Gomes, A.H. Zimerman, preprint IFTP.023/91

[9] O.Babelon and L.Bonora, Phys. Lett 244B(1990)220.

[10] E. Witten Lecture at "Strings 90" Texas A\&M, preprint IASSNS-HEP-90/37(1990).

[11] P. Goddard and D. Olive, Inter. J. Mod. Phys. A1 (1986) 303.

[12] E. Witten, Comm. Math. Phys. 121 (1989), 351.

[13] S.Elitzur, G.Moore, A.Schwimmer and N.Seiberg, Nucl. Phys. B326(1989)108

[14] D. Friedan, E. Martinec, S. Shenker; Nucl. Phys.B271,(1986), 93.

[15] A.M. Polyakov, P.B. Wiegmann; Phys. Lett. 141B, (1984),223. 